\title{
Physical activity and health
}

\section{Even low intensity exercise such as walking is associated with better health}

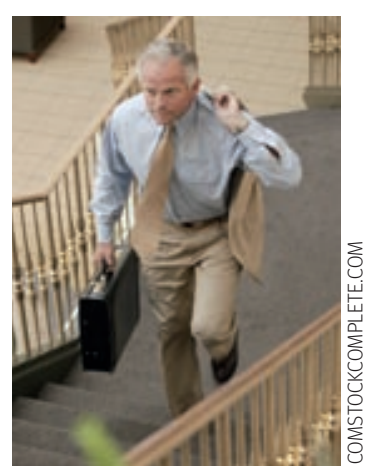

RESEARCH, p 1204

\section{Lars Bo Andersen professor}

Norwegian School of Sport

Sciences, Department of Sports

Medicine, Box 4014, 0806, Oslo,

Norway

Lars.bo.andersen@nih.no

Competing interests: None

declared.

Provenance and peer review:

Commissioned; not externally peer reviewed.

\section{BMJ 2007;334:1173}

doi: 10.1136/bmj.39225.414537.80
Evidence that physical activity improves health is convincing, ${ }^{1}$ but we lack knowledge about how to increase physical activity in individuals and populations. Taking part in sport may improve health, but sport is only taken up by a small proportion of the adult population, and mainly by the better educated.

In this week's $B M J$, a systematic review by Ogilvie and colleagues assesses the effect of interventions to improve walking on how much people walk, physical activity, fitness, disease risk factors, and wellbeing. ${ }^{2}$ It found that interventions tailored to people's needs, which targeted the most sedentary or those motivated to change, can increase walking by up to 30-60 minutes each week. Few studies included in the review assessed clinical benefits from the increased walking, and this remains to be shown in randomised controlled trials.

So what is the evidence so far on the effects of interventions on other types of physical activity? A recent Cochrane review of randomised controlled trials found that trials promoting physical activity in general significantly increased self reported physical activity (standardised mean increase of $0.31,95 \%$ confidence interval 0.12 to 0.50$)$, and fitness $(0.40,0.0 .9$ to 0.70$) .^{3}$ The review by Ogilvie and colleagues also included non-randomised studies, which, although considered weaker forms of evidence, are necessary to assess the effect of population level interventions such as bike lanes, walking paths, and recreational areas.

One non-randomised community intervention in Odense, Denmark, promoted bicycling through many initiatives and increased the number of bicycle trips by more than $20 \%$ over five years. ${ }^{4}$ At the same time, the number of accidents involving cyclists was $20 \%$ lower than in the rest of the country.

Another study found that children who cycled to school were $8 \%$ more fit than children who used other modes of transport including walking. ${ }^{5}$ It concluded that a 10-15 minute session of cycling twice a day would be enough to increase aerobic fitness in children. ${ }^{5}$

Observational studies have consistently shown that children who walk or cycle to school engage in more physical activity (other than the travel activity) than those who travel by other means. ${ }^{6} 7$ This extra activity may reflect selection (children who are generally more active choose active transport) or it may be that children who are encouraged to take up active transport go on to engage in other activities. However, because of the lack of cycle lanes in many countries it may be difficult to promote increased cycling for safety reasons.

A weakness in many of the trials of walking interven- tions is the lack of assessment of health gains; however, epidemiological studies suggest that health benefits of active transport are substantial. The nurses health study found that women who increased both walking distance and speed had a lower risk of cardiovascular disease, type 2 diabetes, and all cause mortality. ${ }^{8} 9$ The risk in the upper quintile of walking was around half that seen in the sedentary group. Similarly, another study found a $30 \%$ lower mortality rate in participants who cycled to work than in non-cyclists after adjusting for general physical activity level, socioeconomic background, and smoking. ${ }^{10}$

Ogilvie and colleagues' study shows that interventions can increase the amount of walking. It has not yet been proved that the lower rates of disease and mortality seen in people who walk is caused by walking itself, but even this low intensity type of exercise probably improves metabolic control and other health parameters. The challenge now is to make politicians work for an environment that promotes walking, and to call on doctors to encourage patients to walk, especially those with disorders such as hypertension, metabolic syndrome, or raised fasting insulin. ${ }^{11}$

1 US Department of Health and Human Services. Physical activity and health: a report of the surgeon general. Atlanta: Centers for Disease Control and Prevention, National Center for Chronic Disease Prevention and Health Promotion, 1997.

2 Ogilvie D, Foster CE, Rothnie H, Cavill N, Hamilton V, Fitzsimons CF, et al; on behalf of the Scottish Physical Activity Research Collaboration (SPARColl). Interventions to promote walking: systematic review. BMJ 2007 doi: $10.1136 /$ bmj.39198.722720.BE.

3 Hillsdon M, Foster C, Thorogood M. Interventions for promoting physical activity. Cochrane Database Syst Rev 2007;(2):CD003180.

4 Troelsen J, Jensen SU, Andersen T. Evaluering afOdense-Danmarks nationale cykelby. Andersen T, Edrén K. Odense: Kerteminde Tryk, 2004.

5 Cooper AR, Wedderkopp N, Wang H, Andersen LB, Froberg K, Page AS. Active travel to school and cardiovascular fitness in Danish children and adolescents. Med Sci Sports Exerc 2006;38:1724-31.

6 Cooper AR, Page AS, Foster LJ, Qahwaji D. Commuting to school: are children who walk more physically active? Am J Prev Med 2003;25:2736.

7 Tudor-Locke C, Ainsworth BE, Adair LS, Popkin BM. Objective physical activity of Filipino youth stratified for commuting mode to school. Med SciSports Exerc 2003;35:465-71.

8 Manson JE, Hu FB, Rich-Edwards JW, Colditz GA, Stampfer MJ, Willett WC, et al. A prospective study of walking as compared with vigorous exercise in the prevention of coronary heart disease in women. N EnglJ Med 1999;341:650-8.

9 Hu FB, Sigal RJ, Rich-Edwards JW, Colditz GA, Solomon CG, Willett WC, et al. Walking compared with vigorous physical activity and risk of type 2 diabetes in women. JAMA 1999;282:1433-9.

10 Andersen LB, Schnohr P, Schroll M, Hein HO. All-cause mortality associated with physical activity during leisure time, work, sports, and cycling to work. Arch Intern Med 2000;160:1621-8.

11 Andersen LB, Boreham CA, Young IS, Davey SG, Gallagher AM, Murray $\mathrm{L}$, et al. Insulin sensitivity and clustering of coronary heart disease risk factors in young adults. The Northern Ireland young hearts study. Prev Med 2006;42:73-7. 


\section{Teaching children basic life support skills}

\section{Improve outcomes but implementation needs to be earlier and more widespread}

\section{RESEARCH, $\mathrm{p} 1201$}

\section{Ian Maconochie}

consultant in paediatric emergency medicine, St Mary's

Hospital, London W2 1NY

ian.maconochie@st-marys.

nhs.uk

Bob Bingham

consultant paediatric anaesthetist The Hospital for Sick Children, Great Ormond Street, London WC1N 3JH

Sheila Simpson

senior resuscitation training officer

Competing interests: None

declared.

Provenance and peer review:

Commissioned; not externally peer reviewed.

BMJ 2007;334:1174

doi: 10.1136/bmi.39218.422650.80
Basic life support performed by bystanders improves outcomes in cardiorespiratory collapse, yet less than $1 \%$ of the general population can perform it effectively. It has been estimated that if $15-20 \%$ of the population could perform basic life support, out of hospital mortality could be significantly reduced. ${ }^{1}$ The most effective way of achieving this is to teach this technique in schools, making it a "life skill."

In this week's $B M J$, a study by Jones and colleagues assesses the effect of a basic life support programme on the ability of children to administer effective chest compressions on a manikin. ${ }^{2}$ Of the three age groups compared (9-10, 11-12, 13-14 years), only children aged over 13 years could perform chest compressions to the recommended depth of 38-51 mm as effectively as adults. However, younger children could place their hands in the correct position on the chest to perform basic life support. The authors suggest that younger children could use this knowledge to instruct an adult on the appropriate technique, despite not being able to do it themselves. Also, young children could be taught how to assess the need for basic life support and activate the emergency medical services. These conclusions support the teaching of basic life support to children.

Structured courses such as the "Injury minimisation programme for schools" (www.impsweb.co.uk), which started in 1994, have integrated the teaching of basic life support into the school curriculum (with the support of local hospitals) and have trained more than 114000 children in the United Kingdom.

Courses are also taught by the British Red Cross, St John's Ambulance Service, St Andrew's Ambulance Service, Heartstart, and Opportunities for Resuscitation and Citizen Safety (ORCS). In Northern Ireland, the "ABC for life" programme was set up in 2005 by the Queen's University Belfast, with the aim of teaching 25000 primary schoolchildren each year. Most of these courses focus on teaching children aged 10 years and older. At this age children are more likely to be developing "abstract thinking" and may be physically capable of performing chest compression.

Basic life support courses can change children's attitudes and behaviour. A large study comparing children

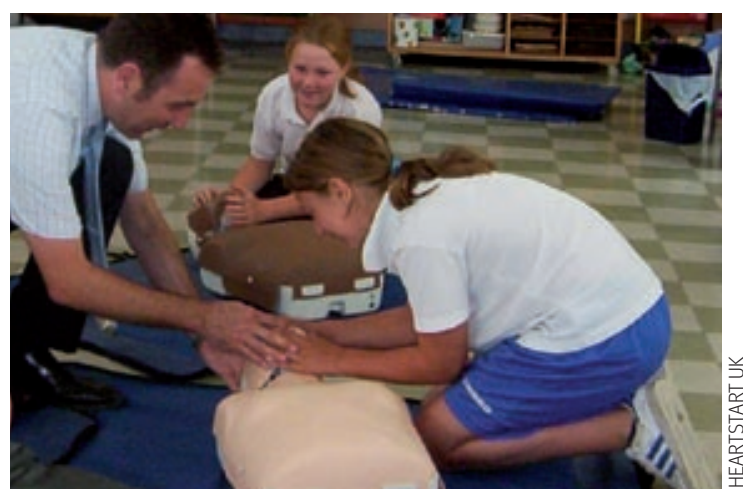

who received such training with those who did not showed that after five months the trained children were more willing to undertake emergency life saving procedures and conducted resuscitation significantly better. ${ }^{3}$

Despite these promising results some caveats exist. In both adults and children the skills decline over time, so refresher courses are needed. A study that repeated the training after six months in school aged children found that knowledge was maintained and that the children's resuscitation skills improved. ${ }^{4}$

"Hands-on" practice is needed to maintain the motor skills required to perform basic life support. Although additional teaching aids such as online resuscitation training may help with the child's knowledge, they do not improve the child's skills. ${ }^{5}$

Effective skills can only be attained through high quality training. ${ }^{6}$ Poor performance arises from inadequate instruction and not allowing sufficient time for the child to learn the technique. This includes time for the method to be demonstrated and for the child to practise the technique under adequate supervision.

High quality teaching can only improve outcomes if uptake is adequate. In countries where teaching basic life support in schools is optional, the uptake of training is low. Barriers include funding and time constraints in the "overfull" school curriculum. ${ }^{7}$ Compulsory training is probably necessary to obtain the levels of skill required to improve outcomes.

The final barrier to implementing basic life support training is lack of resources. Head teachers in Barcelona, sampled in a questionnaire survey, thought that school was the most appropriate setting for teaching these skills, and that such training would increase children's self esteem and could potentially save lives. However, they identified funding as a potential problem, estimating that the cost would be between $€ 5(£ 3.40 ; \$ 6.80)$ and $€ 10$ per child- although this seems a small price to pay for improving survival. ${ }^{9}$

Out of hospital survival from cardiorespiratory collapse could be improved if basic life support was routinely taught to all schoolchildren. Introducing it as early as possible in the school curriculum, perhaps in story and online learning formats, would be non-threatening to young children, who are usually keen to learn and able to absorb new information. Once they are physically able, the transition from theoretical knowledge to practical skills should be relatively easy.

1 Connolly M, Toner P, Connolly D, McCluskey D. The "ABC for life" programme-teaching basic life support in schools. Resuscitation 2007;72:270-9.

2 Jones I, Whitfield R, Colquhoun M, Chamberlain D, Vetter N, Newcombe R. At what age can schoolchildren provide effective chest compressions? An observational study from the Heartstart UK schools training programme. BM/ 2007 doi: 10.1136/bmj.39167.459028.DE.

3 Frederick K, Bixby E, Orzel M, Stewart-Brown S, Willett K. An evaluation of the effectiveness of the injury minimization programme for schools. Inj Prev 2000;6:92-5.

4 Van Kerschaver E, Delooz H, Moens G. The effectiveness of repeated cardiopulmonary resuscitation training in a school population. Resuscitation 1989;3:211-22. 


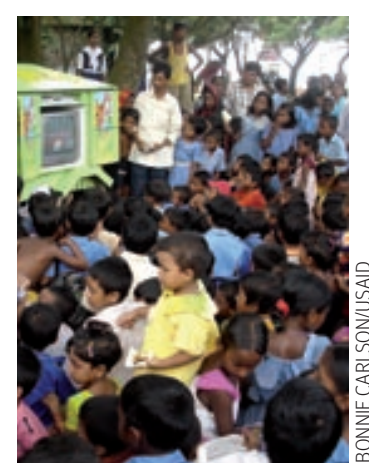

RESEARCH, p 1207

Emilio Perucca professor Clinical Pharmacology Unit, Institute of Neurology, IRCCSC Mondino Foundation, University of Pavia, I-27100 Pavia, Italy perucca@unipv.it

Competing interests: EP has received speaker's or consultancy fees or research grants from the manufacturers of carbamazepine and oxcarbazepine (Novartis); gabapentin, phenytoin, and pregabalin (Pfizer); lamotrigine

(GlaxoSmithKline); levetiracetam (UCB Pharma); tiagabine, valproate, and vigabatrin (Sanofi Aventis); topiramate (Johnson and Johnson); rufinamide and zonisamide (Eisai)

BMJ 2007;334:1175-6 doi: 10.1136/bmj.39065.460208.80

\section{Treatment of epilepsy in developing countries}

\section{Cheap and effective drugs exist but are not accessible to most patients}

Of the 35 million people with epilepsy who live in developing countries, around $85 \%$ receive no treatment at all. ${ }^{12}$ As a consequence, they experience morbidity related to seizures and the psychosocial consequences of stigma and discrimination. Regrettably, most of these people-many of whom are children-could have their seizures completely controlled and they could return to a normal life by taking a single daily dose of a drug that costs less than $\$ 3(£ 1.50 ; € 2.20)$ each year. ${ }^{3}$ In this week's $B M J$, a randomised controlled trial conducted in Bangladesh by Banu and colleagues compares the effects of carbamazepine and phenobarbital on seizure control and behavioural side effects in 108 children with epilepsy. ${ }^{4}$

The World Health Organization recommends phenobarbital as the treatment of choice for partial and tonic clonic seizures in resource restricted countries, ${ }^{5}$ but this policy has been questioned because phenobarbital is thought to be less well tolerated than other antiepileptic drugs. ${ }^{6}$ Concerns apply particularly to children, who are especially vulnerable to this drug's adverse cognitive and behavioural effects. ${ }^{7}$ Differences in tolerability between phenobarbital and other anticonvulsants are probably less prominent than generally thought, however, and they were detected mostly in trials where the assessment of outcomes may have been affected by doctor or patient bias. ${ }^{3} 8$ Importantly, most studies in developing countries did not show excess neuropsychological toxicity of phenobarbital compared with other anticonvulsants, ${ }^{9-11}$ possibly because dosages in these studies tended to be lower than those used in developed countries, or because lack of options make people less willing to report side effects.

The study by Banu and colleagues found no significant difference in behavioural problems such as restlessness and hyperactivity between phenobarbital and carbamazepine ( $7 \% v 11 \%)$, and no significant difference in psychological and behavioural assessments after one year. ${ }^{11}$ Of those children who completed a 12 month follow-up, $47.5 \%$ of those on phenobarbital and $60 \%$ of those on carbamazepine were seizure-free for the last six months.

Conducting clinical trials in resource restricted countries is difficult. As with previous similar studies, the trial by Banu and colleagues has limitations, including an open label design and low power to detect potentially important differences in seizure outcome and behavioural test scores. More children were lost to follow-up in the phenobarbital group (22\%) than in the carbamazepine group (9\%). Therefore, on an intention to treat basis, the proportion of children who were seizure free in the last six months was considerably higher in the carbamazepine group than in the phenobarbital group (50\% v 35\%), which raises questions about potentially lower compliance in children assigned to phenobarbital. Drug concentrations were not reported. The two groups were not well balanced for some characteristics; girls were under-represented in the phenobarbital group, a potentially important factor because behavioural problems were more frequent in girls than in boys.

Despite these limitations, the study shows that most children tolerated phenobarbital well and behaviour even improved in many. This supports other findings in similar settings. In a randomised study of 302 children and adults with epilepsy in rural Kenya, side effects were reported more frequently with phenobarbital than with carbamazepine, but the number of patients with side effects did not differ significantly between drugs; $3 \%$ of patients on phenobarbital were withdrawn for adverse effects and $5 \%$ on carbamazepine. ${ }^{9}$ When 73 children with newly diagnosed epilepsy were randomised to phenobarbital, carbamazepine, or valproate in Taiwan, no significant differences in psychometric scores were found between groups. ${ }^{10}$ Similarly, no treatment related differences in behaviour rating scores were found in 94 children with epilepsy randomised to phenobarbital or phenytoin in rural India. ${ }^{11}$ Observational studies support the conclusion that phenobarbital is relatively well tolerated in developing countries. ${ }^{2}$ Apart from its low cost, phenobarbital has other merits such as efficacy against all seizures other than absences, seizure freedom rates comparable to those associated with modern drugs, a starting dose within the effective range, a low risk of life threatening adverse effects, linear pharmacokinetics, once daily dosing, and availability of a parenteral formulation. ${ }^{8}$

Most controlled trials of phenobarbital in epilepsy have methodological shortcomings, including an open label design, small sample size, and, at times, questionable choice of dosing regimens. ${ }^{3}$ Although larger double blind randomised studies are needed for a better assessment of the role of phenobarbital in the treatment of epilepsy, ${ }^{8}$ Banu and colleagues deserve praise for providing more evidence supporting its use in resource restricted settings.

The burden of untreated epilepsy in terms of human suffering and social costs is enormous. Governments and non-governmental organisations in developing countries need to ensure that effective treatment is available for all. Even in these settings, drug choice should be tailored to the individual, and phenobarbital will not be the best option for all. In fact, the price of drugs is a small part of the cost of ensuring a minimum standard of epilepsy care. Dispensing facilities are often unavailable in remote rural areas, and even when available they often fail to provide a continuous supply of drugs, ${ }^{12}$ which has potentially serious consequences. Seven children in Banu's study discontinued treatment for more than seven days for various 
reasons; four developed convulsive status epilepticus while not taking their drugs. An efficient epilepsy management programme will work only if fully integrated within a community healthcare delivery system, ${ }^{2}$ which should provide not only reliable supplies of drugs, with adequate facilities for storage and dispensing, but also educational programmes for health practitioners and the general population.

1 Kale R. The treatment gap. Epilepsia 2002;43(suppl 6):S31-3. 2 World Health Organization, International Epilepsy Bureau and International League against Epilepsy. Atlas. Epilepsy Care in the World 2005. Geneva: WHO, 2005.

3 Kwan P, Brodie MJ. Phenobarbital for the treatment of epilepsy in the 21st century: a critical review. Epilepsia 2004;45:1141-9.

4 Banu SH, Jahan M, Koli UK, Ferdousi S, Khan NZ, Neville B. Side effects of phenobarbital and carbamazepine in childhood epilepsy: randomised controlled trial. BMJ 2007 doi: 10.1136/ bmj.39022.436389.BE.
5 World Health Organization. Initiative of support to people with epilepsy. Geneva: WHO, 1990 (unpublished document WHO/MNH/ MND/90.3).

6 Michelucci R, Tassinari CA. Phenobarbital, primidone and other barbiturates. In: Shorvon S, Perucca E, Fish D, Dodson E, eds. The treatment of epilepsy. 2nd ed. Oxford: Blackwell, 2004:461-74.

7 Wallace SJ. A comparative review of the adverse effects of anticonvulsants in children with epilepsy. Drug Saf1996;15:378-93.

8 Kale R, Perucca E. Revisiting phenobarbital for epilepsy. BMJ 2004;329:1199-200

9 Feksi AT, Kaamugisha J, Sander JW, Gatiti S, Shorvon SD. Comprehensive primary health care antiepileptic drug treatment programme in rural and semi-urban Kenya. Lancet 1991;337:406-9.

10 Chen Y-J, Kang W-M, So WCM. Comparison of antiepileptic drugs on cognitive function in newly diagnosed epileptic children: a psychometric and neurophysiological study. Epilepsia 1996;37:81-6.

11 Pal DK, Das T, Chaudhury G, Johnson AL, Neville BG. Randomised controlled trial to assess acceptability of phenobarbital for childhood epilepsy in rural India. Lancet 1998;351:19-23.

12 Mac TL, Le VT, Vu AN, Preux PM, Ratsimbazafy V. Antiepileptic drugs availability and professional practices in delivery outlets in a city center in southern Vietnam. Epilepsia 2006;47:330-4.

\title{
Control of methamphetamine misuse
}

\author{
Policy should target substance misuse as a whole, rather than \\ single substances
}

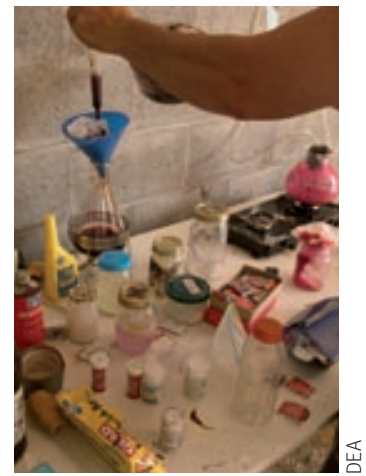

FEATURE, $p 1190$

\section{Tracy D Gunter}

assistant professor of psychiatry Psychiatry Research, University of lowa Carver College of Medicine,

lowa City, lowa 52242, US

tracy-gunter@uiowa.edu

Competing interests: None declared.

Provenance and peer review:

Commissioned; not externally peer reviewed.

BMJ 2007;334:1176-7 doi: 10.1136/bmj.39225.469630.80
Methamphetamine is a highly addictive substance that has caused serious public health problems globally. ${ }^{1}$ As it is relatively easy to manufacture from precursor substances, regulation of precursors has taken centre stage in global strategies for drug control. Recently, the UK Medicines and Healthcare Products Regulatory Agency announced that the precursors pseudoephedrine and ephedrine, also used in flu remedies sold over the counter, may in future be available on prescription only. ${ }^{2}$

Methamphetamine was first synthesised in Japan in 1919 and has been manufactured legally in the United States since the 1950s. Use declined during the 1970s when the public became aware of the harms of amphetamines and practitioners were inhibited from prescribing them by the Controlled Substance Act (1970).http://www.answers.com/topic/ single-convention-on-narcotic-drugs. However, when methamphetamine re-emerged in the 1980 s, it had been transformed into "ice," a smoked form of high purity that produces sustained intoxication. As it exists today, illicit methamphetamine is manufactured in many forms and may be used in many ways (inhaled, ingested, smoked, or injected).

Many definitions of which substances are included in the class of synthetic stimulants or amphetaminetype substances exist, ${ }^{3}$ but generally the class includes amphetamine, methamphetamine, and 3,4 methylenedioxymethamphetamine (MDMA or ecstasy). They cause increased energy, decreased appetite, and a heightened sense of wellbeing. The onset and duration of action vary by specific compound, dose, purity, and route of administration. Complications of use vary greatly and include cardiovascular, neurological, and psychiatric effects. Other possible complications include risk taking behaviour during intoxication and heavy metal exposure as a result of mercuric chloride and lead acetate used in the illicit production of methamphetamine. ${ }^{4}$

According to the World Drug Report issued in 2006 by the United Nations Office of Drugs and Crime, ${ }^{6}$ around 200 million people used illicit drugs. Amphetamine-type substances ranked second, after cannabis, with an estimated 35 million users. Of these, 25 million used amphetamines (including methamphetamine) while the remaining 10 million used ecstasy. When all indicators of amphetamine production and use were combined, the overall global trend was towards a stable to mildly increasing amphetamine market after years of annual increases. However, the results of specific market indicators were mixed, and trends for specific geographical regions varied.

The report also found that seizures of substances diverted for manufacturing amphetamine-type substances reached record levels and exceeded seizures of the end product in $2005 .{ }^{6}$ The main methamphetamine precursors seized were pseudoephedrine and ephedrine. The main amphetamine precursors seized were phenyl-2-propanone and phenylacetic acid. ${ }^{6}$ Although the rate of dismantling laboratories that produce amphetamines has increased, dismantling of large volume international laboratories (so called superlabs) has not.

The relation between the regulation of precursor substances and outcomes in drug users, such as hospital admissions and arrests, has been reported by two studies in the US. ${ }^{7}$ They concluded that regulations limiting access to bulk powder and single ingredient ephedrine and pseudoephedrine products reduced hospital admissions and arrests. However, regulations targeting mixed agent cold remedies used by small scale manufacturers did not result in similar decreases. 
So what is the most effective strategy to reduce harm from amphetamine-type substances? Although the manufacture and misuse of synthetic stimulants contribute greatly to morbidity and mortality in substance users worldwide, the global disease burden of this class of substances is much lower than that of tobacco, alcohol, and marijuana. ${ }^{6}{ }^{10}$ Also, most people who use amphetamine-type substances take multiple substances. ${ }^{11}$

Even if the pattern of drug use is stable over time, drug markets are dynamic. Efforts to prevent the manufacture and use of amphetamine-type substances should, therefore, be integrated into a rational scheme to reduce overall substance use that is designed to tackle existing and emerging drug threats. Overinvesting resources in the control of one drug, or one precursor, carries with it the risk of failing to appreciate emerging threats. For instance, many people fear the "meth crisis," but fewer seem aware of the recent warnings issued by the UN Office of Drugs and Crime about the resurgence of cocaine in Western Europe. ${ }^{12}$

Responses to this crisis should include limiting supply and distribution, ${ }^{13}$ educating the public about harms, screening for early use, and aggressively treating addiction in an integrated approach that tackles addiction in its many forms.
1 Office on Drugs and Crime. Ecstasy and amphetamines: global survey, 2003. New York: ODC, 2003. www.unodc.org/pdf/publications/report ats 2003-09-23 1.pdf.

2 BBC News. Crystal meth fears over medicine. 25 April 2007. http:// news.bbc.co.uk/go/pr/fr/-/1/hi/health/6590513.stm .

3 Maxwell JC. Methamphetamine: a constantly changing epidemic. EpiLink Online Bull 2007;64:22-8.

4 Burton BT. Heavy metal and organic contaminants associated with illicit methamphetamine production. In: Miller MA, ed. Methamphetamine abuse: epidemiologic issues and implications. NIDA research monograph series. Rockville, MD: US Department of Health and Human Services, Public Health Service, Alcohol, Drug Abuse, and Mental Health Services, 1991:47-59. www.nida.nih.gov/pdf/monographs/115.pdf.

5 National Institute on Drug Abuse. Methamphetamine abuse and addiction. Washington, DC: NIDA, 2002 (revised 2006). www.nida.nih. gov/ResearchReports/Methamph/Methamph.html.

6 United Nations Office on Drugs and Crime. World drug report 2006. Vienna: UNODC, 2006. www.unodc.org/unodc/world_drug_report. html.

7 Cunningham JK, Liu LM. Impacts of federal ephedrine and pseudoephedrine regulations on methamphetamine-related hospital admissions. Addiction 2003;98:1229-37.

8 Cunningham JK, Liu LM. Impacts of federal precursor chemical regulations on methamphetamine arrests. Addiction 2005;100:479-88.

9 Thomas G, Davis CG. Comparing the perceived seriousness and actual costs of substance abuse in Canada. Ottawa: Canadian Centre on Substance Abuse, 2007. http://www.ccsa.ca/NR/rdonlyres/98CA9F871BE2-40EB-B345-90984F994BFD/0/ccsa0113502007.pdf.

10 WHO. The tobacco health toll. Cairo: WHO, 2005. www.emro.who.int/ TFl/PDF/TobaccoHealthToll.pdf.

11 Gunter TD, Arndt S, Wenman G. Characteristics of admissions for primary stimulant dependence during 2001. Subst Use Misuse 2006;41: 1277-86.

12 United Nations Office on Drugs and Crime. Annual report 2007. Vienna: UNODC, 2007.www.unodc.org/unodc/annual_report_2007.html.

13 Browenstein HH, Taylor BG. Measuring the stability of illicit drug markets: why does it matter? Drug Alcohol Depend 14 Dec 2006. Epub ahead of print.

\section{Clinical trial registration}

\section{Looking back and moving ahead}

\section{Christine Laine and Richard Horton on behalf of the ICMJE working group}

claine@mail.acponline.org

See bmj.com for full details of the ICMJE working group

Competing interests: Employment: FG was previously editorial director of Current Controlled Trials, which owned the ISRCTN (International Standard Randomised Controlled Trial Number) trials register; SK is employed by the National Library of Medicine, which produces ClinicalTrials.gov, but he is not responsible for activities or policies regarding ClinicalTrials.gov. Expert testimony: FG. Other: RH is co-chair and JMD and HCS are members of the WHO ICTRP scientific advisory group; MBVDW is a member of the government advisory committee for the Australian and New Zealand Clinical Trials Registry.

PS's affiliation as a representative and past president of the World Association of Medical Editors

(WAME) does not imply endorsement by WAME member journals that are not part of the ICMJE.
In 2005, the International Committee of Medical Journal Editors (ICMJE) initiated a policy requiring investigators to deposit information about trial design into an accepted clinical trials registry before the onset of patient enrolment. ${ }^{1}$ This policy aimed to ensure that information about the existence and design of clinically directive trials was publicly available, an ideal that leaders in evidence based medicine have advocated for decades. ${ }^{2}$ The policy precipitated much angst among research investigators and sponsors, who feared that registration would be burdensome and would stifle competition. Yet, the response to this policy has been overwhelming. The ICMJE promised to re-evaluate the policy two years after implementation. Here, we summarise that re-evaluation, specifically commenting on registries that meet the policy requirements, the types of studies that require registration, and the registration of trial results. As is always the case, the ICMJE establishes policy only for the 12 member journals (a detailed description of the ICMJE and its purpose is available at www.icmje. org), but many other journals have adopted our initial trial registration recommendations, and we hope that they will also adopt the modifications discussed in this update.

The research community has embraced trial registration. Before the ICMJE policy, ClinicalTrials.gov, the largest trial registry at the time, contained 13153 trials; this number climbed to 22714 one month after the policy came into effect. ${ }^{3}$ In April 2007, the registry contained over 40000 trials, with more than 200 new trial registrations occurring weekly (D Zarin, personal communication). The four other registries that meet the ICMJE criteria have also grown as scores of journals have adopted the ICMJE clinical trials registration policy. In response to burgeoning registration, many investigators, sponsors, and government agencies have asked the ICMJE to recognise their local registries as databases that meet the policy. Fortunately, the World Health Organization's (WHO) International Clinical Trials Registry Platform (ICTRP), which was nascent when the ICMJE began to require trial registration, has matured rapidly and provides options for those who desire a wider array of registries. The ICTRP has taken the first steps towards developing a network of primary and partner registers that meet WHO specified criteria. ${ }^{4}$ Primary registers are WHO selected registers managed by not-for-profit entities that will accept registrations for any interventional trials, delete duplicate entries from their own register, and provide data directly to WHO. Partner registers, which will be more numerous, will include registers that submit data to primary registers but limit their own register to trials 
Summary

- In addition to accepting registration in any of the five existing registries, the International Committee of Medical Journal Editors (ICMJE) will accept registration of clinical trials in any of the primary registers that participate in WHO's International Clinical Trials Registry Platform (ICTRP). Registration in a partner register only is insufficient

- The ICMJE will begin to implement the WHO definition of clinical trials for all trials that begin enrollment on or after 1 July 2008. This definition states that a clinical trial is "any research study that prospectively assigns human participants or groups of humans to one or more healthrelated interventions to evaluate the effects on health outcomes"

- The ICMJE will not consider results posted in the same clinical trials registry in which the primary registration resides to be a previous publication if the results are presented in the form of a brief, structured ( $(500$ words) abstract or table.

in a restricted area (such as a specific disease, company, academic institution, or geographic region).

The ICMJE strongly supports WHO's efforts, through the ICTRP, to develop a coordinated process for identifying, gathering, deduplicating, and searching trials from registries around the world, thus eventually providing a one stop search portal for those seeking information about clinical trials. In addition to the five existing registries, the ICMJE will now also accept registration in any of the primary registers that participate in WHO ICTRP. Because it is crucial that trial registries are independent of for-profit interests, the ICMJE policy requires registration in a WHO primary register rather than solely in a partner register, since for-profit entities manage some partner registers. As previously, trial registration with missing or uninformative fields for the minimum data elements is inadequate. ${ }^{1}$

Initially, the ICMJE required registration of all clinically directive trials, which it defined as "any research project that prospectively assigns human subjects to intervention or comparison groups to study the cause and effect relationship between a medical intervention and a health outcome." ${ }^{1}$ In May 2005, the ICMJE clarified this definition to exclude preliminary trials designed to study pharmacokinetics or major unknown toxicity (phase I trials). ${ }^{5}$ However, the ICMJE recognises the potential benefit of having information about preliminary trials in the public domain, because these studies can guide future research or signal safety concerns. Consequently, the ICMJE is expanding the definition of the types of trials that must be registered to include these preliminary trials and adopts WHO's definition of a clinical trial, "any research study that prospectively assigns human participants or groups of humans to one or more health-related interventions to evaluate the effects on health outcomes." ${ }^{4}$ Health related interventions include any intervention used to modify a biomedical or health related outcome (for example, drugs, surgical procedures, devices, behavioural treatments, dietary interventions, and process of care changes). Health outcomes include any biomedical or health related measures obtained in patients or participants, including pharmacokinetic measures and adverse events. As previously, purely observational studies (those in which the assignment of the medical intervention is not at the discretion of the investigator) will not require registration. The ICMJE member journals will start to implement the expanded definition of clinically directive trials for all trials that begin enrollment on or after 1 July 2008 . Those who are uncertain whether their trial meets the expanded ICMJE definition should err on the side of registration if they wish to seek publication in an ICMJE journal.

Over the time during which registration of trial methods has become common practice, several forces have begun advocating for registration of trial results. We recognise that the climate for results registration will probably change dramatically and unpredictably over coming years. For the present, the ICMJE will not consider results posted in the same primary clinical trials register in which the initial registration resides as previous publications if the results are presented in the form of a brief, structured ( $<500$ words) abstract or table. The ICMJE favours a standard abstract format for results reporting, and the CONSORT (Consolidated Standards for the Reporting of Trials) group's forthcoming guidelines for abstracts related to trials may be one such option. The ICMJE believes that parties interested in results registration should consider requiring the deposition of such an abstract in the registry 24 months after closure of data collection if results are not published in a peer reviewed venue by that time. The registered abstract should either cite any related full, peer reviewed publications or include a statement that indicates that the report has not yet been published in a peer reviewed journal. Researchers should be aware that editors may consider more detailed deposition of trial results in publicly available registries to be prior publication. When submitting a paper, authors should fully disclose to editors all posting in registries of results of the same or closely related work.

Three years ago, trial registration was the exception; now it is the rule. Registration facilitates the dissemination of information among clinicians, researchers, and patients, and it helps to assure trial participants that the information that accrues as a result of their altruism will become part of the public record. WHO's global efforts towards comprehensive trial registration and the ICMJE's requirements for registration aim to increase public trust in medical science.

1 Clinical trial registration: a statement from the International Committee of Medical Journal Editors. www.icmje.org/clin trial.pdf.

2 Simes RJ. Publication bias: the case for an international registry of clinical trials. J Clin Oncol 1986;4:1529-41.

3 Zarin DA, Tse T, Ide NC. Trial registration at ClinicalTrials.gov between May and October 2005. N Engl J Med 2005;353:2779-87.

4 World Health Organization. International Clinical Trials Registry Platform. www.who.int/ictrp/about/details/en/index.html.

5 International Committee of Medical Journal Editors. Is this clinical trial fully registered? A statement from the International Committee of Medical Journal Editors. www.icmje.org/clin trialup.htm. 